\title{
Existence and continuity for the $\varepsilon$-approximation equilibrium problems in Hadamard spaces
}

\section{Pakkapon Preechasilp*}

\section{"Correspondence:}

preechasilpp@gmail.com

Program in Mathematics, Faculty of

Education, Pibulsongkram Rajabhat

University, Phitsanulok, Thailand

\section{Introduction}

Let $K$ be a nonempty subset of a linear space $X$ and $f: K \times K \rightarrow \mathbb{R}$ a bifunction. The equilibrium problem, for short (EP), is defined as follows:

Find $x \in K$ such that $f(x, y) \geq 0, \quad \forall y \in K$.

(EP) was formulated by Blum and Oettli [1] in 1994. It is shown in the paper that (EP) includes many classical problems in optimization, such as variational inequality, constrained minimization, fixed point, Nash-equilibrium problems, etc. Since that time, there has been extensive study in various directions; see [2-4] and references therein. Almost all extended and generalized results are studied in linear spaces. Actually, some applications in optimization problems cannot be considered in linear space. Recently, in the past decade, many optimization problems were investigated on nonlinear space, such as Riemannian manifolds, hyperbolic space and geodesic spaces, for example in [5-7]. In 2012, Colao et al. [8] first established the existence for an equilibrium point in the setting of Hadamard manifolds. By using an analogous KKM lemma, the authors developed an equilibrium theory and gave applications to optimization-related problems in Hadamard manifolds. This paper is an inspiration for studying the generalized and/or application of (EP) in Hadamard manifolds; see $[5,9,10]$. The KKM lemma is the main tool for proving an existence result for (EP). The proof of the KKM lemma relies on the fact that the closed convex hull of any finite set is compact. It was mentioned by Kopecká and Reich [11] that, in any Hadamard 
spaces, the closed convex hull of a finite number of points might not be compact. In order to extend [8]'s results to Hadamard spaces, Niculescu and Roventa [12] first introduced the famous KKM lemma and proved the existence of a minimax point in a Hadamard space which has the convex hull finite property. However, there are few papers, e.g. [13, 14], which deal with the existence for the solution of (EP) in Hadamard spaces.

On the other hand, when we have a mathematical problem, not only the existence of the solution for that problem but also the stability for the solution set of the problem is investigated. Roughly speaking this is about how a slight change in a parameter of a given mathematical problem could affect the solution or solution set of the problem. Hence, stability may be understood as lower (upper) semicontinuity, continuity, and Lipschitz or Hölder continuity. Currently, such a study is, in fact, very important as many useful mathematical problems are usually approximately solved by the problem. Since data of mathematical models of practical problems are obtained by measuring devices or statistical records, the models are also approximations, and hence their exact solutions are the acceptability of the approximate solution, based on a certain allowed error on the parameters of the problem. However, concerning continuity, results of approximate solutions to the parametric equilibrium problems, e.g. [15-17], are established in linear spaces.

Motivated and inspired by the above literature, the aim of this paper is to establish the existence result for (EP) and continuity of the solution mapping to parametric $\varepsilon$-approximate parametric equilibrium problem, for short (PEP), in the setting of Hadamard spaces. We also give applications to constrained minimization problems and Nash-equilibrium problems.

\section{Preliminaries}

Let $(X, d)$ be a metric space. $X$ is called a Hadamard space if it complete and if for each pair of points $x, y \in X$ there exists a point $w \in X$ such that for all $z \in X$

$$
d^{2}(z, w) \leq \frac{1}{2} d^{2}(z, x)+\frac{1}{2} d^{2}(z, y)-\frac{1}{4} d^{2}(x, y)
$$

A Hadamard space is sometimes called a global nonpositive curvature space or complete CAT(0) space. In the rest of the paper, we denote by $X$ the Hadamard space. It is well known that, in Hadamard spaces, for each $x, y \in X$ there exists a unique point $z$ in the geodesic segment joining $x$ to $y$ with

$$
d(z, x)=t d(x, y) \quad \text { and } \quad d(z, y)=(1-t) d(x, y) .
$$

We also denote by $[[x, y]]$ the geodesic segment joining $x$ to $y$, that is, $[[x, y]]=\{(1-t) x \oplus t y$ : $t \in[0,1]\}$. A subset $C$ of $X$ is convex if $[[x, y]] \subseteq C$ for all $x, y \in C$.

Let $A \subseteq X$ be a nonempty subset. Then the convex hull of $A$, denoted $c o(A)$, is defined as the intersection of all convex subset containing $A$. It is easy to see that $\operatorname{co}(A)=\bigcup_{n=0}^{\infty} A_{n}$, where $A_{0}=A$, and for $n \geq 1$, the set $A_{n}$ is the union of all geodesics with start and end in $A_{n-1}$. The convex hull of a finite subset is not necessarily closed, and likewise the closed convex hull of $A$, denoted $\overline{c o}(A)$, is the intersection of all closed convex subsets containing $A$. It is shown in [18] that if $A_{1}, \ldots, A_{n}$ are compact convex subsets in a locally convex Hausdorff space, then the convex hull of their union is also compact. 
Definition 1 A function $g: C \rightarrow \mathbb{R}$ is called geodesic convex if the function $g \circ c:[0,1] \rightarrow$ $\mathbb{R}$ is convex for each geodesic $c:[0,1] \rightarrow X, c(t)=c_{t}$, i.e.,

$$
g\left(c_{t}\right) \leq \operatorname{tg}\left(x_{1}\right)+(1-t) g\left(x_{2}\right)
$$

for all $t \in(0,1)$. The function $g$ is called geodesic concave if $-g$ is geodesic convex.

We recall the KKM mapping in the setting of Hadamard spaces which is used for the existence result for $(\mathbf{E P})$.

Definition 2 [12] Let $C$ be a nonempty subset of a Hadamard space $X$. A multivalued mapping $G: C \rightrightarrows E$ is said to be a KKM mapping if

$$
c o(F) \subseteq \bigcup_{x \in F} G(x)
$$

for every nonempty finite subset $F$ of $C$.

The following concept of the convex hull finite property was first introduced by [12] and used to prove the analog of the KKM lemma in Hadamard spaces.

Definition 3 [12] We say that a Hadamard space $X$ has the convex hull finite property if the closed convex hull of every nonempty finite family of points of $X$ has the fixed point property.

Lemma 4 [12] Suppose that $X$ is a Hadamard space with the convex hull finite property and $C$ is a nonempty subset of $X$. Furthermore, suppose $T: X \rightrightarrows X$ is a KKM mapping with closed values. Then, if $T(z)$ is compact for some $z \in C$, then

$$
\bigcap_{x \in C} T(x) \neq \emptyset
$$

Throughout this paper, if not otherwise specified, let $X$ be a Hadamard space, $M$ be metric space, and $A \subseteq X$ be nonempty set.

Let $N\left(\mu_{0}\right) \subset M$ be a neighborhood of the considered point $\mu_{0}$. Let $K: M \rightrightarrows A$ be a nonempty set-valued mapping and $f: A \times A \times M \rightarrow \mathbb{R}$.

For each $\mu \in N\left(\mu_{0}\right)$, we consider the following parametric equilibrium problem (PEP): Find $x \in K(\mu)$ such that

$$
f(x, y, \mu) \geq 0, \quad \forall y \in K(\mu) .
$$

Let $S(\mu)$ be the efficient solution set of (2.1), that is,

$$
S(\mu):=\{x \in K(\mu): f(x, y, \mu) \geq 0, \forall y \in K(\mu)\} .
$$

For each $\mu \in N\left(\mu_{0}\right)$ and $\varepsilon \geq 0$, let $\widetilde{S}(\varepsilon, \mu)$ denote the set of $\varepsilon$-approximate solution set to (2.1), that is,

$$
\widetilde{S}(\varepsilon, \mu):=\{x \in K(\mu): f(x, y, \mu)+\varepsilon \geq 0, \forall y \in K(\mu)\} .
$$


We collect some concepts and properties of semicontinuity for set-valued mappings in metric spaces.

Definition $5[19,20]$ Let $X$ and $Y$ be topological spaces, $T: X \rightrightarrows Y$ be a set-valued mapping.

(i) $T$ is said to be upper semicontinuous (u.s.c., for short) at $x_{0} \in X$ iff for any open set $V$ containing $T\left(x_{0}\right)$, there exists an open set $U$ containing $x_{0}$ such that $T(x) \subseteq V$ for all $x \in U$.

(ii) $T$ is said to be lower semicontinuous (l.s.c., for short) at $x_{0} \in X$ iff for any open set $V$ with $T\left(x_{0}\right) \cap V$, there exists an open set $U$ containing $x_{0}$ such that $T(x) \cap V \neq \emptyset$ for all $x \in U$.

(iii) $T$ is said to be continuous at $x_{0} \in X$ iff it is both l.s.c. and u.s.c.

Proposition $6[19,21]$ Let $X$ and $Y$ be metric spaces and $T: X \rightrightarrows Y$ and $x_{0} \in X$. Then the following are equivalent:

(i) $T(\cdot)$ is l.s.c. at $x_{0}$;

(ii) if $\left\{x_{n}\right\}$ is any sequence such that $x_{n} \rightarrow x_{0}$ and $G \subseteq Y$ an open subset such that $T\left(x_{0}\right) \cap G \neq \emptyset$, then

$$
\exists N \geq 1: T\left(x_{n}\right) \cap G \neq \emptyset, \quad \forall n \geq N
$$

(iii) if $\left\{x_{n}\right\}$ is any sequence such that $x_{n} \rightarrow x_{0}$ and $y_{0} \in T\left(x_{0}\right)$ arbitrary, then there is a sequence $\left\{y_{n}\right\}$ with $y_{n} \in T\left(x_{n}\right)$ such that $y_{n} \rightarrow y_{0}$.

Proposition $7[19,21]$ Let $X$ and $Y$ be metric spaces and $T: X \rightrightarrows Y$ and $x_{0} \in X$. Then the following are equivalent:

(i) $T(\cdot)$ is u.s.c. at $x_{0}$;

(ii) if $x_{0} \in X$ and $\left\{x_{n}\right\}$ is any sequence such that $x_{n} \rightarrow x_{0}$ and $V \subseteq Y$ an open subset such that $T\left(x_{0}\right) \subseteq V$, then

$$
\exists N \geq 1: F\left(x_{n}\right) \subseteq V, \quad \forall n \geq N
$$

Let $A,\left\{A_{n}\right\}_{n \in \mathbb{N}}$ be nonempty sets in a metric space $X$. We shall use the following notations:

$$
\begin{aligned}
& \liminf A_{n}=\left\{x \in X: \exists\left\{x_{n}\right\} \text { such that } x_{n} \in A_{n}, \forall n \in \mathbb{N}, x_{n} \rightarrow x\right\} \\
& \lim \sup A_{n}=\left\{x \in X: \exists\left\{n_{k}\right\} \text { and } \exists\left\{x_{n_{k}}\right\} \text { such that } x_{n_{k}} \in A_{n_{k}}, \forall k \in \mathbb{N}, x_{n_{k}} \rightarrow x\right\} .
\end{aligned}
$$

Remark 8 It follows from (iii) in Proposition 6 that $T(\cdot)$ is l.s.c. at $x_{0}$ iff, for every sequence $x_{n} \rightarrow x_{0}$, we have $T\left(x_{0}\right) \subseteq \liminf T\left(x_{n}\right)$.

\section{Existence and continuity results}

In this section, we first present the existence result for an $\varepsilon$-approximate solution for (EP). We also study the continuity of $\varepsilon$-approximate solution maps for (PEP).

Theorem 9 Let $K$ be a nonempty closed convex subset of a Hadamard space $X$ which has the convex hull finite property. For any given $\varepsilon>0$, let $f: K \times K \rightarrow \mathbb{R}$ be a bifunction such that 
(i) for any $x \in K, f(x, x) \geq 0$;

(ii) for every $x \in K$, the set $\{y \in K: f(x, y)+\varepsilon<0\}$ is convex set;

(iii) for every $y \in K, f(\cdot, y)$ is upper semicontinuous;

(iv) there exist a compact set $L \subseteq X$ and a point $y_{0} \in L \cap K$ such that

$$
f\left(x, y_{0}\right)+\varepsilon<0, \quad \forall x \in K \backslash L .
$$

Then there exists a point $x_{0} \in L \cap K$ such that

$$
f\left(x_{0}, y\right)+\varepsilon \geq 0, \quad \forall y \in K
$$

Proof Define the mapping $G: K \rightrightarrows K$ as follows:

$$
G(y)=\{x \in K: f(x, y)+\varepsilon \geq 0\} \quad \text { for all } y \in K .
$$

We first prove that $G(y)$ is closed for all $y \in K$. Let $\left\{x_{m}\right\}$ be a sequence in $G(y)$ such that $x_{m} \rightarrow x^{\prime}$ for some $x^{\prime} \in X$. Then we have $f\left(x_{m}, y\right)+\varepsilon \geq 0$ for all $m$. It follows from (iii) that

$$
f\left(x^{\prime}, y\right)+\varepsilon \geq \limsup _{m \rightarrow \infty} f\left(x_{m}, y\right)+\varepsilon \geq 0
$$

This implies that $G(y)$ is closed for all $y \in K$. By condition (iv) there exists a point $y_{0} \in K$ for which $G\left(y_{0}\right) \subseteq L$. Since $L$ is compact, we see that $G\left(y_{0}\right)$ is also. We want to prove that $G(\cdot)$ is a KKM mapping. We prove this by contradiction, suppose that $G$ is not a KKM mapping. Then there exist a finite subset $A=\left\{x_{1}, x_{2}, \ldots, x_{n}\right\}$ of $K$ and a point $\hat{x} \in \operatorname{co}(A)$ such that $\hat{x} \notin G\left(y_{i}\right)$ for all $i=1, \ldots, n$. Thus

$$
f\left(\hat{x}, y_{i}\right)+\varepsilon<0, \quad \forall i \in\{1,2, \ldots, n\}
$$

That is, for any $i \in\{1, \ldots, n\}$,

$$
y_{i} \in\{y \in K: F(\hat{x}, y)+\varepsilon<0\} .
$$

Since the set $\{y \in K: F(\hat{x}, y)+\varepsilon<0\}$ is convex, we get

$$
\hat{x} \in \operatorname{co}\left(\left\{y_{1}, y_{2}, \ldots, y_{n}\right\}\right) \subseteq\{y \in K: f(\hat{x}, y)+\varepsilon<0\},
$$

which is a contradiction to the assumption (i). Then by Lemma 4 , there exists a point $x_{0} \in K$ such that

$$
x_{0} \in \bigcap_{y \in K} G(y) .
$$

Hence, there exists $x_{0} \in L \cap K$ such that

$$
f\left(x_{0}, y\right)+\varepsilon \geq 0, \quad \forall y \in K
$$




\section{Remark 10}

(i) Our Theorem 9 is closely related to Theorem 3.3 in [13], which is presented in the sense of a minimax inequality.

(ii) If $\varepsilon=0$, then we have the existence result for an exact solution for (EP) which encloses the result in [12].

By setting $L=K$ in Theorem 9, the following corollary is immediately obtained.

Corollary 11 Let $K$ be a nonempty compact convex subset of a Hadamard space $X$ which has the convex hull finite property. For any given $\varepsilon>0$, let $f: K \times K \rightarrow \mathbb{R}$ be a bifunction such that

(i) for any $x \in K, f(x, x) \geq 0$;

(ii) for every $x \in K$, the set $\{y \in K: f(x, y)+\varepsilon<0\}$ is convex set;

(iii) for every $y \in K, f(\cdot, y)$ is upper semicontinuous.

Then there exists a point $x_{0} \in K$ such that

$$
f\left(x_{0}, y\right)+\varepsilon \geq 0, \quad \forall y \in K
$$

The following example shows that the $\varepsilon$-approximate solution for (EP) depends on $\varepsilon$. Consider $\mathbb{R}^{2}$ with the usual Euclidean meter $d(\cdot, \cdot)$ and $\|\cdot\|$, which are defined by

$$
d(x, y)=\|x-y\|=\sqrt{\left(x_{1}-y_{1}\right)^{2}+\left(x_{2}-y_{2}\right)^{2}},
$$

where $x=\left(x_{1}, x_{2}\right)$ and $y=\left(y_{1}, y_{2}\right)$. We define the radial metric $d_{r}$ by

$$
d_{r}(x, y)= \begin{cases}d(x, y), & \text { if } y=t x \text { for some } t \in \mathbb{R} ; \\ d(x, 0)+d(y, 0), & \text { otherwise. }\end{cases}
$$

Then $X:=\left(\mathbb{R}^{2}, d_{r}\right)$ is an $\mathbb{R}$-tree with radial meter $d_{r}$.

Example 12 Let $X$ be an $\mathbb{R}$-tree with radial meter $d_{r}$. We put $K=[[(1,0),(0,1)]]$ and $f(x, y)=\left(x_{1}+x_{2}\right)-0.3$. After calculating we easily get

$$
\widetilde{S}(\varepsilon, \mu)= \begin{cases}{[[(0,0.3),(0,1)]] \cup[[(0.3,0),(1,0)]],} & \text { if } 0 \leq \varepsilon<0.3 \\ {[[(1,0),(0,1)]],} & \text { if } \varepsilon \geq 0.3 .\end{cases}
$$

The following corollary is a sufficient condition for the existence of the parametric $\varepsilon$ approximate solution (PEP).

Corollary 13 For any given $\varepsilon>0$, assume that there exists a neighborhood $N\left(\mu_{0}\right)$ of $\mu_{0}$ satisfying the following conditions:

(i) for each $\mu \in N\left(\mu_{0}\right), K(\mu)$ is a nonempty, compact and convex valued;

(ii) for each $\mu \in N\left(\mu_{0}\right)$ and each $x \in K\left(N\left(\mu_{0}\right)\right), f(x, x, \mu) \geq 0$;

(iii) for each $x \in K\left(N\left(\mu_{0}\right)\right)$, the set $\left\{y \in K\left(N\left(\mu_{0}\right)\right): f(x, y, \mu)+\varepsilon<0\right\}$ is convex set;

(iv) for each $\mu \in N\left(\mu_{0}\right)$ and each $y \in K\left(N\left(\mu_{0}\right)\right), f(\cdot, y, \mu)$ is upper semicontinuous on $K\left(N\left(\mu_{0}\right)\right)$.

Then for each $\mu \in N\left(\mu_{0}\right), \widetilde{S}(\varepsilon, \mu)$ is nonempty and compact. 
Now, we give the sufficient conditions for continuity of an approximate solution $\widetilde{S}$ at $\left(\varepsilon_{0}, \mu_{0}\right)$. Relying on the existence theorem for $\varepsilon$-approximation (EP), we assume that the solution of the $\varepsilon$-approximation exists for all $(\varepsilon, \mu) \in \mathbb{R}_{+} \cup\{0\} \times M$.

Theorem 14 Consider the $\varepsilon$-approximate (PEP). We assume that the following conditions hold:

$\left(\mathrm{C}_{1}\right) K$ is continuous at $\mu_{0}$ and $K\left(\mu_{0}\right)$ has compact and convex valued;

$\left(C_{2}\right)$ there exists a neighborhood $N\left(\mu_{0}\right)$ of $\mu_{0}$ such that $f(\cdot, \cdot, \cdot)$ is continuous on $K\left(N\left(\mu_{0}\right)\right) \times$ $K\left(N\left(\mu_{0}\right)\right) \times\left\{\mu_{0}\right\}$

$\left(C_{3}\right)$ for each $y \in K\left(\mu_{0}\right), f\left(\cdot, y, \mu_{0}\right)$ is a geodesic concave function on $K\left(N\left(\mu_{0}\right)\right)$.

Then $\widetilde{S}(\cdot, \cdot)$ is l.s.c. at $\left(\varepsilon_{0}, \mu_{0}\right)$.

Proof We first prove that $\widetilde{S}\left(\cdot, \mu_{0}\right)$ is l.s.c. at $\varepsilon_{0}$. Suppose not, then there exist an open set $G \subseteq X$ and a sequence $\left\{\varepsilon_{n}\right\}$ with $\varepsilon_{n} \rightarrow \varepsilon_{0}$ such that

$$
\widetilde{S}\left(\varepsilon_{0}, \mu_{0}\right) \cap G \neq \emptyset \text { but } \widetilde{S}\left(\varepsilon_{n}, \mu_{0}\right) \cap G=\emptyset \text { for all } n \in \mathbb{N} \text {. }
$$

Note that $\widetilde{S}\left(\varepsilon_{1}, \mu_{0}\right) \subseteq \widetilde{S}\left(\varepsilon_{2}, \mu_{0}\right)$, if $\varepsilon_{1} \leq \varepsilon_{2}$.

We claim that $\varepsilon_{0}>\varepsilon_{n}$ for all $n \in \mathbb{N}$. If not, there exists $\bar{N} \in \mathbb{N}$ such that $\varepsilon_{0} \leq \varepsilon_{\bar{N}}$. That is,

$$
\widetilde{S}\left(\varepsilon_{0}, \mu_{0}\right) \subseteq \widetilde{S}\left(\varepsilon_{\bar{N}}, \mu_{0}\right), \quad \text { and so } \emptyset \neq \widetilde{S}\left(\varepsilon_{0}, \mu_{0}\right) \cap G \subseteq \widetilde{S}\left(\varepsilon_{\bar{N}}, \mu_{0}\right) \cap G \text {, }
$$

which is a contradiction to (3.1). So we have the claim.

Let $x_{0} \in \widetilde{S}\left(0, \mu_{0}\right)$ and $\bar{x} \in \widetilde{S}\left(\varepsilon_{0}, \mu_{0}\right) \cap G$. We set $x_{n}=\frac{\varepsilon_{0}-\varepsilon_{n}}{\varepsilon_{0}} \bar{x} \oplus \frac{\varepsilon_{n}}{\varepsilon_{0}} x_{0}$, then $x_{n} \rightarrow \bar{x}$ as $n \rightarrow \infty$. Since $\bar{x} \in G$, there exists $n_{0} \in \mathbb{N}$ such that

$$
\frac{\varepsilon_{0}-\varepsilon_{n_{0}}}{\varepsilon_{0}} \bar{x} \oplus \frac{\varepsilon_{n_{0}}}{\varepsilon_{0}} x_{0}=x_{n_{0}} \in G
$$

We claim that $x_{n_{0}} \in \widetilde{S}\left(\varepsilon_{n_{0}}, \mu_{0}\right)$. Since $x_{0} \in \widetilde{S}\left(0, \mu_{0}\right)$ and $\bar{x} \in \widetilde{S}\left(\varepsilon_{0}, \mu_{0}\right)$, we have, for all $y \in$ $K\left(\mu_{0}\right)$,

$$
f\left(x_{0}, y, \mu_{0}\right) \geq 0 \text { and } f\left(\bar{x}, y, \mu_{0}\right)+\varepsilon_{0} \geq 0 .
$$

Hence

$$
\frac{\varepsilon_{0}-\varepsilon_{n_{0}}}{\varepsilon_{0}} f\left(x_{0}, y, \mu_{0}\right) \geq 0 \quad \text { and } \quad \frac{\varepsilon_{n_{0}}}{\varepsilon_{0}} f\left(\bar{x}, y, \mu_{0}\right)+\frac{\varepsilon_{n_{0}}}{\varepsilon_{0}} \varepsilon_{0} \geq 0 .
$$

It follows from (iii) that

$$
f\left(\frac{\varepsilon_{0}-\varepsilon_{n_{0}}}{\varepsilon_{0}} \bar{x} \oplus \frac{\varepsilon_{n_{0}}}{\varepsilon_{0}} x_{0}, y, \mu_{0}\right) \geq \frac{\varepsilon_{0}-\varepsilon_{n_{0}}}{\varepsilon_{0}} f\left(x_{0}, y, \mu_{0}\right)+\frac{\varepsilon_{n_{0}}}{\varepsilon_{0}} f\left(\bar{x}, y, \mu_{0}\right)+\varepsilon_{n_{0}} \geq 0 .
$$

Therefore, $x_{n_{0}} \in \widetilde{S}\left(\varepsilon_{n_{0}}, \mu_{0}\right)$, which leads to a contradiction to (3.1). We can conclude that $\widetilde{S}\left(\cdot, \mu_{0}\right)$ is l.s.c. at $\varepsilon_{0}$. 
Next, we prove that $\widetilde{S}(\varepsilon, \cdot)$ is l.s.c. at $\mu_{0}$ for all $\varepsilon>0$. By the assumption of nonemptiness of the exact solution, we set the following strict $\varepsilon$-approximate solution maps:

$$
\widetilde{S}_{S}(\varepsilon, \mu):=\left\{x \in K\left(\mu_{0}\right): f\left(x, y, \mu_{0}\right)+\varepsilon>0, \forall x \in K\left(\mu_{0}\right)\right\} .
$$

It is clear that, for any $\mu \in N\left(\mu_{0}\right)$,

$$
\emptyset \neq \widetilde{S}(0, \mu) \subseteq \widetilde{S}_{S}(\varepsilon, \mu) \subseteq \widetilde{S}(\varepsilon, \mu)
$$

We show that $\widetilde{S}_{S}(\varepsilon, \cdot)$ is l.s.c. at $\mu_{0}$ for all $\varepsilon>0$. If not, there are $\bar{x} \in \widetilde{S}_{S}\left(\varepsilon, \mu_{0}\right)$ and a sequence $\mu_{n} \rightarrow \mu_{0}$, but for all $x_{n} \in \widetilde{S}_{S}\left(\varepsilon, \mu_{n}\right), x_{n} \nrightarrow \bar{x}$. Since $K\left(\mu_{0}\right)$ is l.s.c. at $\mu_{0}$, there exists $\bar{x}_{n} \in$ $K\left(\mu_{n}\right)$ with $\bar{x}_{n} \rightarrow \bar{x}$. By the above contradiction assumption, there must be a subsequence $\left\{\bar{x}_{n_{k}}\right\}$ of $\left\{\bar{x}_{n}\right\}$ such that $\bar{x}_{n_{k}} \notin \widetilde{S}_{S}\left(\varepsilon, \mu_{n}\right)$, i.e., there exists $y_{n_{k}} \in K\left(\mu_{n_{k}}\right)$ such that

$$
f\left(\bar{x}_{n_{k}}, y_{n_{k}}, \mu_{n_{k}}\right)+\varepsilon \leq 0 .
$$

Since $K(\cdot)$ is u.s.c. at $\mu_{0}$ and $K\left(\mu_{0}\right)$ is compact valued, there exists $\bar{y} \in K\left(\mu_{0}\right)$ such that $y_{n_{k_{j}}} \rightarrow \bar{y}$. From (3.4) and continuity of $f(\cdot, \cdot, \cdot)$ on $K\left(N\left(\mu_{0}\right)\right) \times K\left(N\left(\mu_{0}\right)\right) \times\left\{\mu_{0}\right\}$, we get

$$
f\left(\bar{x}, \bar{y}, \mu_{0}\right)+\varepsilon \leq 0
$$

which leads to a contradiction. Hence $\widetilde{S}_{S}(\varepsilon, \cdot)$ is l.s.c. at $\mu_{0}$.

Next, we claim that

$$
\widetilde{S}\left(\varepsilon, \mu_{0}\right) \subseteq \operatorname{cl}\left(\widetilde{S}_{S}\left(\varepsilon, \mu_{0}\right)\right)
$$

For any $x_{0} \in \widetilde{S}\left(\varepsilon, \mu_{0}\right)$ and $x_{1} \in \widetilde{S}_{S}\left(\varepsilon, \mu_{0}\right)$, we put $x_{t}=(1-t) x_{0} \oplus t x_{1}, t \in(0,1)$. Then $x_{t} \rightarrow x_{0}$ as $t \rightarrow+0$. Since $f\left(\cdot, y, \mu_{0}\right)$ is geodesic concave, we have

$$
\begin{aligned}
f\left(x_{t}, y, \mu_{0}\right)+\varepsilon & \geq(1-t) f\left(x_{0}, y, \mu_{0}\right)+t f\left(x_{1}, y, \mu_{0}\right)+\varepsilon \\
& =(1-t)\left(f\left(x_{0}, y, \mu_{0}\right)+\varepsilon\right)+t\left(f\left(x_{1}, y, \mu_{0}\right)+\varepsilon\right) \\
& \geq 0, \quad \forall y \in K\left(\mu_{0}\right) .
\end{aligned}
$$

Hence, $x_{t} \in \widetilde{S}_{S}\left(\varepsilon, \mu_{0}\right)$. This implies that $x_{0} \in \operatorname{cl}\left(\widetilde{S}_{S}\left(\varepsilon, \mu_{0}\right)\right)$. So, we have the claim. Hence, for all $\varepsilon>0, \widetilde{S}_{S}(\varepsilon, \cdot)$ is l.s.c. at $\mu_{0}$. Thus, for any $\mu_{m} \rightarrow \mu_{0}$

$$
\operatorname{cl}\left(\widetilde{S}_{S}\left(\varepsilon, \mu_{0}\right)\right) \subseteq \liminf \widetilde{S}_{S}\left(\varepsilon, \mu_{m}\right)
$$

Combining the last inequality with (3.3) and (3.5), we have

$$
\widetilde{S}\left(\varepsilon, \mu_{0}\right) \subseteq \operatorname{cl}\left(\widetilde{S}_{S}\left(\varepsilon, \mu_{0}\right)\right) \subseteq \liminf \widetilde{S}_{S}\left(\varepsilon, \mu_{m}\right) \subseteq \liminf \widetilde{S}\left(\varepsilon, \mu_{m}\right)
$$

Therefore $\widetilde{S}(\varepsilon, \cdot)$ is l.s.c. at $\mu_{0}$. We can conclude from Step 1 and 2 that $\widetilde{S}(\cdot, \cdot)$ is l.s.c. at $\left(\varepsilon_{0}, \mu_{0}\right)$.

Theorem 15 Assume that the conditions $\left(\mathrm{C}_{1}\right)-\left(\mathrm{C}_{2}\right)$ hold. Then $\widetilde{S}(\cdot, \cdot)$ is u.s.c. at $\left(\varepsilon_{0}, \mu_{0}\right)$. 
Proof We prove by contradiction, suppose that $\widetilde{S}(\cdot, \cdot)$ is not u.s.c. at $\left(\varepsilon_{0}, \mu_{0}\right)$. Then there exist an open neighborhood $U$ of $\widetilde{S}\left(\varepsilon_{0}, \mu_{0}\right)$ and a sequence $\left\{\left(\varepsilon_{n}, \mu_{n}\right)\right\}$ converging to $\left(\varepsilon_{0}, \mu_{0}\right)$ such that

$$
\widetilde{S}\left(\varepsilon_{n}, \mu_{n}\right) \nsubseteq \mathcal{U}, \quad \forall n \in \mathbb{N} .
$$

Then there exists some $x_{n} \in \widetilde{S}\left(\varepsilon_{n}, \mu_{n}\right)$ such that

$$
x_{n} \notin U, \quad \forall n \in \mathbb{N} .
$$

Since $x_{n} \in \widetilde{S}\left(\varepsilon_{n}, \mu_{n}\right)$, we have $x_{n} \in K\left(\mu_{n}\right)$. By the assumption, $K(\cdot)$ is u.s.c. being compact valued at $\mu_{0}$, then we see that there exists a subsequence $\left\{x_{n_{k}}\right\}$ such that $x_{n_{k}} \rightarrow x^{*}$.

We will show that $x^{*} \in \widetilde{S}\left(\varepsilon_{0}, \mu_{0}\right)$. Suppose to the contrary that $x^{*} \notin \widetilde{S}\left(\varepsilon_{0}, \mu_{0}\right)$. Then there exists $y^{*} \in A\left(\mu_{0}\right)$ such that

$$
f\left(x^{*}, y^{*}, \mu_{0}\right)+\varepsilon<0 .
$$

Since $K(\cdot)$ is l.s.c. at $\mu_{0}$ and $y^{*} \in K\left(\mu_{0}\right), \varepsilon_{n} \rightarrow \varepsilon_{0}$ and $\mu_{n} \rightarrow \mu_{0}$, we see that there exists $y_{n} \in \widetilde{S}\left(\varepsilon_{n}, \mu_{n}\right)$ such that $y_{n} \rightarrow y^{*}$. It follows from $y_{n} \in K\left(\mu_{n}\right)$ that

$$
f\left(x_{n}, y_{n}, \mu_{n}\right)+\varepsilon \geq 0, \quad \forall n \in \mathbb{N} .
$$

Condition $\left(\mathrm{C}_{2}\right)$ gives $f\left(x^{*}, y^{*}, \mu_{0}\right)+\varepsilon \geq 0$. This is a contradiction with (3.7). Hence, we have $x^{*} \in \widetilde{S}\left(\varepsilon_{0}, \mu_{0}\right) \subseteq U$. Since $x_{n} \rightarrow x^{*}$ and $U$ is an open set, there exists some $n_{0} \in \mathbb{N}$ such that $x_{n} \in U$ for all $n \geq n_{0}$, which is a contradiction with (3.6). Therefore, $\widetilde{S}(\cdot, \cdot)$ is u.s.c. at $\left(\varepsilon_{0}, \mu_{0}\right)$.

Theorem 16 Consider the $\varepsilon$-approximate $(\boldsymbol{P E P})$. We assume that the conditions $\left(\mathrm{C}_{1}\right)-\left(\mathrm{C}_{3}\right)$ hold. Then $\widetilde{S}(\cdot, \cdot)$ is continuous at $\left(\varepsilon_{0}, \mu_{0}\right)$.

The following example illustrates that Theorem 16 cannot apply with exact solution maps to (PEP).

Example 17 Let $X$ be an $\mathbb{R}$-tree with radial meter $d_{r}$. We put $M=[0,1]$ and $K(\mu)=$ $[[(1,0),(0,1)]]$ and $f(x, y, \mu)=-\mu\left(x_{1}+x_{2}\right)$. Then the assumptions of Theorem 9 are satisfied. Direct computations give us the approximate solution set, for positive $\varepsilon>0$ and $\mu \in M$,

$$
\widetilde{S}(\varepsilon, \mu)= \begin{cases}{[[(1,0),(0,1)]],} & \text { if } \mu=0, \\ {\left[\left[\left(\min \left\{\frac{\varepsilon}{\mu}, 1\right\}, 0\right),\left(0, \min \left\{\frac{\varepsilon}{\mu}, 1\right\}\right)\right]\right],} & \text { if } \mu \neq 0 .\end{cases}
$$

We see that $\widetilde{S}(\cdot, \cdot)$ is not l.s.c. at $(0,0)$. Indeed, for $\left(\varepsilon_{n}, \mu_{n}\right)=\left(\frac{1}{2 n}, \frac{1}{n}\right) \rightarrow(0,0)$ and $(1,0) \in$ $\widetilde{S}(0,0)$, it is clear that there is no sequence in $\widetilde{S}\left(\frac{1}{2 n}, \frac{1}{n}\right)=\left[\left[\left(\frac{1}{2}, 0\right),\left(0, \frac{1}{2}\right)\right]\right]$ which converges to $(1,0)$. Hence, $\widetilde{S}(\cdot, \cdot)$ is not l.s.c. at $(0,0)$. 


\section{Applications}

As mentioned in Section 1, the (EP) contains many optimization related problems as special cases. Therefore, we derive the continuity of the results of Section 3 for such special cases. In this section, we give applications to constrained minimization problems and Nash-equilibrium problems.

\subsection{Constrained minimization problems}

Let $X, M, A$, and $K$ be as in Section 3. Let $g: A \times M \rightarrow \mathbb{R}$. For $\mu \in M$, we consider the constrained minimization problem, for short (CMP), as follows:

Finding $x \in K(\mu)$ such that $g(x, \mu) \leq g(y, \mu), \quad \forall y \in K(\mu)$.

If we set $f(x, y, \mu)=g(y, \mu)-g(x, \mu)$, we see that (CMP) becomes an example of (EP). For $(\varepsilon, \mu) \in \mathbb{R}_{+} \times M$, we denote the parametric $\varepsilon$-approximate solution set of (CMP) by $\widetilde{S}_{C M P}(\varepsilon, \mu)$, that is,

$$
\widetilde{S}_{C M P}(\varepsilon, \mu)=\{x \in K(\mu) \text { such that } g(y, \mu)-g(x, \mu)+\varepsilon \geq 0, \forall y \in K(\mu)\} .
$$

Now, we present the continuity result for $(\mathbf{C M P})$; we assume that $\widetilde{S}_{C M P}(\varepsilon, \mu)$ exists for all $(\varepsilon, \mu) \in \mathbb{R}_{+} \cup\{0\} \times M$.

Corollary 18 Assume that the following conditions hold:

$\left(\mathrm{M}_{1}\right) K$ is continuous at $\mu_{0}$ and $K\left(\mu_{0}\right)$ is compact and convex;

$\left(\mathrm{M}_{2}\right)$ there exists a neighborhood $N\left(\mu_{0}\right)$ of $\mu_{0}$ such that $g$ is continuous in $K\left(N\left(\mu_{0}\right)\right) \times\left\{\mu_{0}\right\}$;

$\left(\mathrm{M}_{3}\right) g\left(\cdot, \mu_{0}\right)$ is geodesic convex in $K\left(N\left(\mu_{0}\right)\right)$.

Then $\widetilde{S}_{C M P}(\cdot, \cdot)$ is continuous at $\left(\varepsilon_{0}, \mu_{0}\right)$.

Proof It suffices to show that all assumptions in Theorem 16 hold. It is easy to see that $\left(\mathrm{C}_{1}\right)$ and $\left(\mathrm{C}_{2}\right)$ hold. We only check that $\left(\mathrm{C}_{3}\right)$ holds. For any $x_{1}, x_{2} \in K\left(N\left(\mu_{0}\right)\right), t \in[0,1]$ and $y \in K\left(N\left(\mu_{0}\right)\right)$, we put $x_{t}=t x_{1} \oplus(1-t) x_{2}$. Then

$$
\begin{aligned}
& f\left(x_{t}, y, \mu_{0}\right)-t f\left(x_{1}, y, \mu_{0}\right)-(1-t) f\left(x_{2}, y, \mu_{0}\right) \\
&= g\left(y, \mu_{0}\right)-g\left(x_{t}, \mu_{0}\right)-t\left(g\left(y, \mu_{0}\right)-g\left(x_{1}, \mu_{0}\right)\right)-(1-t)\left(g\left(y, \mu_{0}\right)-g\left(x_{2}, \mu_{0}\right)\right) \\
&= g\left(y, \mu_{0}\right)-t g\left(x_{1}, \mu_{0}\right)-(1-t) g\left(x_{2}, \mu_{0}\right) \\
&-t\left(g\left(y, \mu_{0}\right)-g\left(x_{1}, \mu_{0}\right)\right)-(1-t)\left(g\left(y, \mu_{0}\right)-g\left(x_{2}, \mu_{0}\right)\right) \\
&= t g\left(x_{1}, \mu_{0}\right)+(1-t) g\left(x_{2}, \mu_{0}\right)-g\left(x_{t}, \mu_{0}\right) \geq 0 .
\end{aligned}
$$

This implies that $\left(C_{3}\right)$ holds. This completes the proof.

\subsection{Nash-equilibrium problems}

Let $I=\{1,2, \ldots N\}$ be a finite index set which denotes the set of players. For any $i \in I$, consider $X_{i}$ to be a Hadamard space where the strategy set $K_{i} \subseteq X_{i}$ of the $i$ th player will be given. Let $K=\prod_{i=1}^{N} K_{i}$ belong to the product Hadamard space $X=\prod_{i=1}^{N} X_{i}$. For arbitrary $x=$ $\left(x_{1}, \ldots, x_{N}\right) \in X$ we define $x^{i}=\left(x_{1}, \ldots, x_{i-1}, x_{i+1}, \ldots, x_{N}\right)$. Suppose that for every $i \in I$ there 
exists a payoff function $f_{i}: K \rightarrow \mathbb{R}$ representing the loss of each player, depending on the strategies of all player. A Nash equilibrium [22] is a strategy profile with the property that the strategy of each player is an optimal choice given the strategy of the other players. Thus, a strategy $x=\left(x_{1}, x_{2}, \ldots, x_{N}\right)$ is a Nash equilibrium if

$$
f_{i}(x) \leq f_{i}\left(x^{i}, y_{i}\right), \quad \forall y_{i} \in K_{i} .
$$

This problem can be formulated as an equilibrium problem by defining the bifunction $F: K \times K \rightarrow \mathbb{R}$ as

$$
F(x, y)=\sum_{i \in I}\left(f_{i}\left(x^{i}, y_{i}\right)-f_{i}(x)\right), \quad \forall(x, y) \in K \times K .
$$

It can be shown that $x$ is a Nash-equilibrium point if and only if it is an a solution of the equilibrium problem (4.3). Our aim is to consider the continuity for Nash-equilibrium points, we assume further that the strategy set $K_{i}$ and payoff function $f_{i}$ are perturbed, that is, they depend on a perturbation parameter $\mu$ of a metric space $M$ for all $i$. More precisely, $f_{i}: K \times M \rightarrow \mathbb{R}$ and $K_{i}: M \rightrightarrows X$ for all $i$.

For $(\varepsilon, \mu) \in \mathbb{R}_{+} \times M$, we denote the $\varepsilon$-approximate solution set of Nash-equilibrium points by $\widetilde{S}_{N E P}(\varepsilon, \mu)$, that is,

$$
\widetilde{S}_{N E P}(\varepsilon, \mu)=\{x \in K(\mu) \text { such that } F(x, y)+\varepsilon \geq 0, \forall y \in K(\mu)\} .
$$

\section{Corollary 19 We assume that the following conditions hold.}

$\left(\mathrm{N}_{1}\right) K_{i}(\cdot)$ is continuous at $\mu_{0}$ and $K_{i}\left(\mu_{0}\right)$ is compact and convex valued for all $i$;

$\left(\mathrm{N}_{2}\right)$ there exists a neighborhood $N\left(\mu_{0}\right)$ of $\mu_{0}$ such that $f_{i}(\cdot, \cdot, \cdot)$ is continuous on $K_{i}\left(N\left(\mu_{0}\right)\right) \times$ $K_{i}\left(N\left(\mu_{0}\right)\right) \times\left\{\mu_{0}\right\}$ for all $i$;

$\left(\mathrm{N}_{3}\right)$ for each $y \in K_{i}\left(N\left(\mu_{0}\right)\right), f_{i}\left(\cdot, y, \mu_{0}\right)$ is a geodetically concave function on $K_{i}\left(N\left(\mu_{0}\right)\right)$ for all $i$.

Then $\widetilde{S}_{\text {NEP }}(\cdot, \cdot)$ is continuous at $\left(\varepsilon_{0}, \mu_{0}\right)$.

Proof If we set $K(\mu)=\prod_{i=1}^{N} K_{i}(\mu)$ and $F$ as in (4.3), we see that all assumptions in Theorem 16 are satisfied. Hence, we have the desired conclusion.

\section{Conclusions}

In this paper, the classical existence result for $\varepsilon$-approximate equilibrium problems is proved in the framework of the Hadamard space (nonlinear space). We also establish the sufficient conditions for the continuity of $\varepsilon$-approximate solution maps to equilibrium problems. However, the conclusion of Theorem 16 is not true for an exact solution, in the case $\varepsilon=0$. As applications, we apply the continuity results to constrained minimization problems and Nash-equilibrium problems. 


\section{Acknowledgements}

This research was supported by Pibulsongkram Rajabhat University grant RDI-2-58-3-12. The author is grateful to the anonymous referees for their helpful comments and suggestions, which improved the presentation of this manuscript.

Received: 5 October 2015 Accepted: 19 April 2016 Published online: 03 May 2016

\section{References}

1. Blum, E, Oettli, W: From optimization and variational inequalities to equilibrium problems. Math. Stud. 63, 123-145 (1994)

2. Chen, B, Huang, NJ: Continuity of the solution mapping to parametric generalized vector equilibrium problems. J. Glob. Optim. 56, 1515-1528 (2012)

3. Li, XB, Li, SJ: Existence of solutions for generalized vector quasiequilibrium problems. Optim. Lett. 4, 17-28 (2010)

4. Hai, NX, Khanh, PQ: The existence of $\varepsilon$-solutions to general quasiequilibrium problems. Vietnam J. Math. 35(4), 563-572 (2007)

5. Kristály, A: Nash-type equilibria on Riemannian manifolds: a variational approach. J. Math. Pures Appl. 101, 660-688 (2014)

6. Udriste, C: Convex Functions and Optimization Methods on Riemannian Manifolds, vol. 597. Kluwer Academic, Dordrecht (1994)

7. Sturm, KT: Probability measures on metric spaces of nonpositive curvature. In: Auscher, P, et al. (eds.) Heat Kernels and Analysis on Manifolds, Graphs, and Metric Spaces. Cont. Math., vol. 338, pp. 357-390 (2003)

8. Colao, V, López, G, Marino, G, Márquez, M: Equilibrium problems in Hadamard manifolds. J. Math. Anal. Appl. 338(1), 61-77 (2012)

9. Zhou, L-W, Huang, N-J: Existence of solutions for vector optimization on Hadamard manifolds. J. Optim. Theory Appl. 157, 44-53 (2013)

10. Chaipunya, P, Kumam, P: Nonself KKM maps and corresponding theorems in Hadamard manifolds. Appl. Gen. Topol. 16(1), 37-44 (2015)

11. Kopecká, E, Reich, S: Nonexpansive retracts in Banach spaces. Banach Cent. Publ. 11, 161-174 (2007)

12. Niculescu, CP, Roventa, L: Fan's inequality in geodesic spaces. Appl. Math. Lett. 22, 1529-1533 (2009)

13. Shabanian, S, Vaezpour, SM: A minimax inequality and its applications to fixed point theorems in CAT(0) spaces. Fixed Point Theory Appl. 2011, 61 (2011)

14. Lu, H, Lu, D, Hu, Q, Yuan, G: Fixed point theorems in CAT(0) spaces with applications. J. Inequal. Appl. (2014). doi:10.1186/1029-242X-2014-320

15. Li, XB, Li, SJ: Continuity of approximate solution mapping for parametric equilibrium problems. J. Glob. Optim. 51, 541-548 (2011)

16. Wangkeeree, R, Boonman, P, Preechasilp, P: Lower semicontinuity of approximate solution mappings for parametric generalized vector equilibrium problems. J. Inequal. Appl. (2014). doi:10.1186/1029-242X-2014-421

17. Anh, LQ, Tam, TN: Hausdorff continuity of approximate solution maps to parametric primal and dual equilibrium problems. Top (2015). doi:10.1007/s11750-015-0390-z

18. Day, MM: Normed Linear Spaces. Ergebnisse Der Mathematik Und Iher Grenzgebiete. Springer, New York (1973)

19. Aubin, JP, Ekeland, I: Applied Nonlinear Analysis. Wiley, New York (1984)

20. Aubin, JP, Frankowska, H: Set-Valued Analysis. Birkhäuser, Boston (1990)

21. Berge, C: Topological Spaces. Oliver \& Boyd, London (1963)

22. Nash, J: Non-cooperative games. Ann. Math. 54(2), 286-295 (1951)

\section{Submit your manuscript to a SpringerOpen ${ }^{\circ}$ journal and benefit from:}

- Convenient online submission

Rigorous peer review

- Immediate publication on acceptance

- Open access: articles freely available online

- High visibility within the field

- Retaining the copyright to your article 